\title{
Food Choice Behaviors of Lactating Women: Association with Body Mass Index and Fruits and Vegetables Intake in Central Amhara Region, Ethiopia-An Observational Study
}

\author{
Gesessew Kibr \\ Department of Food and Nutritional Sciences, Faculty of Agriculture, Shambu Campus, Wollega University, Shambu, Ethiopia \\ Correspondence should be addressed to Gesessew Kibr; gesessewkibr@gmail.com
}

Received 29 November 2020; Revised 2 February 2021; Accepted 10 February 2021; Published 28 February 2021

Academic Editor: Abdah Md Akim

Copyright (c) 2021 Gesessew Kibr. This is an open access article distributed under the Creative Commons Attribution License, which permits unrestricted use, distribution, and reproduction in any medium, provided the original work is properly cited.

\begin{abstract}
Background. What food people choose to eat is embraced by circumstances that are essentially influenced by the underlying motives of food choices which are important to a healthful dietary change. Therefore, this study aimed to explore food choice behaviors associated with nutritional status and FVs intake in women during lactation from central Amhara. Methods. A multistage sampling technique was applied to select study participants. A study was conducted among 423 randomly selected lactating women using a face-to-face interview of the questionnaire. A community-based cross-sectional, quantitative study with descriptive and analytical components was done. Anthropometrics measurements such as weight, height, waist, and hip circumference were taken from all participants. Body mass index was calculated to determine the nutritional status of women. Data were analyzed using SPSS version 20 computer software program. Association between dependent and independent variables was identified by logistic regression analysis, and a $P$-value $<0.05$ was taken as statistically significant. Results. About $21.7 \%$ of women were underweight. Approximately $37.4 \%$ of women had good consumption trends to fruits and vegetables. Healthy meal eating motivation, price-consciousness, and mood concern were identified as the most significant behaviors of food choice, influencing the nutritional status of women with AOR (95\% CI) of 2.1 (1.21-3.62), 3.01 (1.32-6.9), and 0.5 (0.30-0.95), respectively. Natural content (AOR (95\%): 2.37 (1.39-4.06)), mood concern (AOR (95\%): 2.29 (1.15-4.56)), religion concern (AOR (95\%): 2.45 (1.37-4.4)), husband encouragement (AOR (95\%): 2.26 (1.04-4.89)), and availability of milk and milk products (AOR (95\%): 1.6 (1.39-2.74)) and fruits and vegetables (AOR (95\%): $1.66(1.16-3.48)$ ) in home were associated significantly with a good intake of fruit and vegetables. Conclusions. This finding provides a useful insight into the food choice motivations of a group of women with preference for natural foods, mood concern, religion concern, and husband support with good fruit and vegetable intake. This can help practitioners make recommendations for health promotion strategies. Emphasis on assisting women with healthy meal eating and avoiding psychological stress is important. Nutrition education about healthy food choice is recommended for communities.
\end{abstract}

\section{Introduction}

Malnutrition from preventable causes excessively affects the wellbeing of millions of people, especially women and children under five years old in developing countries [1]. A change in lifestyle and working conditions apart from changing the dietary pattern hugely attributes to nutritionrelated noncommunicable chronic diseases (NCDs) in developing countries too [2-5]. In Ethiopia, studies reported malnutrition in women like overweight/obesity [4, 6-9], undernutrition $[4,6,7,10-13]$, micronutrient deficiencies
[14], anemia and vitamin A deficiency [11], and diet-related NCDs burden [12, 15-17]. Chronic health disorders such as obesity, diabetes, and cardiovascular diseases have been increasing in Ethiopia for the last few decades [18]. Human healthy dietary intakes are dependent on consuming enough variety of foods to provide all the required nutrients to sustain life. If food systems do not provide adequate diversity of foods to meet these needs constantly, malnutrition could be prevalent among populations [19].

Improving nutrition across the life span of the population especially reproductive-age women is essential for 
the well-being of families and communities and the successful economic and social advancement [2-4, 12-15, 19]. According to the Ethiopian Demographic and Health Survey, $27 \%$ of Ethiopian women are too thin, with $6 \%$ being overweight or obese [12]. According to the Federal Minister of Health $(\mathrm{FMOH}), 17 \%$ of women were anemic and $6 \%$ of women were experiencing vitamin A deficiency [11]. In Ethiopia, different studies reported the prevalence of undernutrition among women $[6,7,9,12]$, which was about $38 \%, 27 \%, 25 \%$, and $17.4 \%$, respectively.

According to the USAID report on Ethiopia nutrition using 2012 data, $27 \%$ of women were subjected to micronutrient deficiencies [14]. Dietary behavior like intake of a diet low in fruits and vegetables and high in sodium contributes significantly to the noncommunicable disease burden in Ethiopia [17]. A cross-sectional study in Gilgel Gibe found that $9 \%$ of the population was affected by chronic diseases [16]. The Ethiopian nationwide study reported a strong association between nutritional impairment and the development of chronic diseases such as cardiovascular diseases, cancer, and diabetes [15]. The proportion of noncommunicable disease deaths associated with low fruit consumption slightly increased from $11.3 \%$ in 1990 to $11.9 \%$ in 2013 [17]. Consumption of fruits and vegetables (FVs) is the most sustainable way of controlling micronutrient deficiencies in poor-resource communities and associated with reduced risk of health conditions/noncommunicable diseases such as obesity, diabetes, cancer, and cardiovascular diseases $[19,20]$. Since they are rich in vitamins, minerals, and dietary fiber and low in calories [21], the micronutrient supplies of FVs are vital for the functioning of the gastrointestinal tract as they enable the body to use other nutrients (fats and carbohydrates) required for its normal function [22].

Based on the evidence of the role of FVs in the prevention of many health problems, the Food and Agriculture Organization (FAO) and World Health Organization (WHO) have recommended that people should eat at least $400 \mathrm{~g}$ of FVs per person per day or five servings a day; a standard portion size is assumed to be $80 \mathrm{~g}$. However, actual portions tend to be lower for vegetables and higher for fruits, at least two servings $(160 \mathrm{~g})$ of fruits and three servings $(240 \mathrm{~g})$ of vegetables with at least one serving of vegetables involving dark green and leafy or orange vegetable (nutrientrich vegetable) or about $146 \mathrm{~kg}$ per capita (per person) per year FVs combined (excluding potatoes and other starchy tubers, cassava, and sugar). However, the consumption of FVs in African countries ranged from $27 \mathrm{~kg}$ to $114 \mathrm{~kg}$ per person per year, far below the WHO/FAO minimum recommended levels. Ethiopia stands out as a country with low consumption of FVs, both in urban and rural areas with an average intake of fewer than 100 grams per day [23]. In this setting, identifying drivers of food choice associated with the actual food consumption is basic to guide the nutritional status of the target population in a healthier direction [24].

Poor dietary behavior like a diet low in FVs and high in sodium contributes significantly to the noncommunicable disease burden in Ethiopia [19]. A cross-sectional study done in Gilgel Gibe found that $9 \%$ of the population is affected by chronic diseases [18]. The Ethiopian nationwide study reported a strong association between nutritional impairment and the development of chronic diseases such as cardiovascular diseases, cancer, and diabetes [16].The proportion of noncommunicable diseases and deaths associated with low fruit consumption slightly increased from $11.3 \%$ in 1990 to $11.9 \%$ in 2013 [17].

Nutrition-related health problems are among the Ethiopian government's priorities, especially towards the attainment of the sustainable development goals, to end hunger, food insecurity, and all other forms of malnutrition $[10,11,15]$. Such low achievements could be further explained by poor food choice decisions, which remain poorly understood and resulting in focusing on less healthy foods which is a silent critical issue contributing to overall malnutrition in Ethiopia [4, 14-17]. Women are the most crucial group to produce productive and effective human power so that they should remain in a healthy status [25]. Adequate nutrition by maintaining optimal food choices throughout a woman's time and healthy lifestyle behavior is a significant consideration to improve the health of both babies and women [23-26]. Lactating women are considered as a nutritionally vulnerable group and subjected to nutritional stress due to their nursing process [27]. This physiological state increases the feeling of hunger which makes them different from other women of reproductive age like pregnant women who had a unique physiological status and nonpregnant and lactating women. Lactating women have additional nutritional recommendations [28]. Because of this nature, lactating women may have different food choice behavior compared to other childbearing aged women. In the past, several studies were conducted on food consumption patterns of lactating women in Ethiopia [7, 9, 29]. Furthermore, food choice behaviors are rapidly evolving in response to recent changes from different perspectives in the communities' livelihoods and cultural norms in the face of nutrition transition and other trends. However, no studies have been conducted for a deeper understanding of the key drivers of food choice associated with food consumption patterns and nutritional status in that nutritionally vulnerable group. Therefore, this study was designed to explore various determinants of food choice associated with nutritional status and FVs consumption among women in Ethiopia.

\section{Materials and Methods}

2.1. Study Setting, Design, and Period. The study was conducted in Debre Berhan Town, North Shewa Zone, Amhara Regional State, Ethiopia. Debre Berhan is a zonal town and located in central Ethiopia at a distance of $130 \mathrm{Km}$ away from Addis Ababa and $695 \mathrm{Km}$ away from Bahir Dar which is the regional capital. Its astronomical location is $11^{\circ} 06^{\prime}$ North Latitude and $39^{\circ} 45^{\prime}$ East Longitude. The topography is classified as $86 \%$ flat, $10 \%$ sloppy, and $4 \%$ mountainous. The total area of the city is 21169.95 hectares of land [15].

The dominant economic activities in the town are tanneries, blanket factories, and beverage factories. Agriculture in the suburbs provides livelihood to a large section of 
society. In the town, there is one government university, various colleges, schools, banks, hospitals, both public and private, various clinics, and primary, secondary, and preparatory schools. Based on the population projection from the central statistics agency of Ethiopia (2015), the town has a total population of 102,500 [30]. The majority (94.12\%) of the inhabitants practiced Ethiopian Orthodox Christianity [15].

The study area was selected since it is the scarcity of researched information regarding the driver of food choice in addition to resources limitation by the researcher. All participants completed a short background questionnaire. Descriptively, the cross-sectional study design was employed to investigate the characteristics of women, FVs consumption, and specific food choice behaviors from Debre Berhan town between March and June 2016. Based on the following criteria, study participants were recruited:

Lactating women aged 15-49 years

Permanent residents of Debre Berhan town for at least six months before data collection

Willing to participate in the study

The following criteria were set for ruling out the study participants:

Lactating women who were severely ill and unable to communicate with the data collectors

Not willing to participate in the study

Those who could not be found at home after three visits were excluded from the study

2.2. Sample Size Determination. The base sample size was calculated for each objective by using the StatCalc application of Epi Info TM 7.0.8.3 (2011) as follows:

For the First Objective. The determination of base sample size was done by using Epi Info based on the assumption that considered an anticipated population proportion of $50 \%$ for drivers of food choice with a $95 \%$ confidence level and a $5 \%$ margin of error.

For the Second Objective. From a study conducted in the Republic of Ireland, sex and age had shown a sociodemographic association with healthy value consideration during food choice. Accordingly, women were more concerned about the health value in choosing foods than men with an AOR of 2.68 and $37.6 \%$ of men (unexposed subjects) had a healthy eating motivation. Besides, compared to 18-35 years' aged participants, 51-64 and >64 years' aged participants were more concerned with the health value of foods during food choice with AOR of 2.7 and 6.03. Furthermore, 23.7\% of $51-64$ and $17.7 \%$ of $>64$ aged groups had the healthy eating motivation (unexposed subjects) [31]. Using Epi Info and taking $95 \%$ confidence level, $80 \%$ power, $1: 1$ ratio of the unexposed group and exposed group, outcomes in unexposed groups, and given odds ratios, base sample sizes for those assumptions were calculated as follows. From the calculated base sample sizes, 384 was the largest and used to calculate the final sample size by adjusting the nonresponse rate of $10 \%$.

Final sample size $=[$ largest base sample size + (largest base sample size* nonresponse rate)]

$n=[(384+(384 * 0.10))]=423$

2.3. Sampling Procedures. The detailed schematic representation of sampling techniques of the study is presented in Figure 1 below. Debre Berhan town is purposefully selected as a study area and has 9 kebeles. To get representative study samples, all kebeles were used to select study participants. Before the actual data collection, preregistration of lactating women in each kebele was done to count the numbers of lactating women. In all kebeles, the households with lactating women were identified through the house-to-house visit by ketene leaders. In this exercise, a total of 1087 lactating women were registered from the nine kebeles of Debre Berhan town. After house-to-house visit, a sampling frame or a complete list of lactating women was prepared for each kebele. The lottery method was applied to the selected lactating woman from a household that contains more than one lactating woman. Then, the total sample size (423) was proportionally allocated to the number of each kebele's lactating women who were found during preregistration.

To select those proportionally allocated lactating women from each kebele, the simple random sampling technique was applied. Random selections of proportionally allocated lactating women were done by using the planning application of ENA for the SMART computer software program (2007). In doing this, first, a random number table was generated for each kebele based on the given range and required a random number (number of proportionally allocated lactating women) from the range. Using these generated specific random numbers, the corresponding lactating women were marked from the complete list as study participants in each kebele of Debre Berhan town.

2.4. Data Collection Method and Instrument. Most important data were collected using an interviewer-administered questionnaire using three trained female data collectors and two supervisors. The interview was conducted at home and it took, on average, 20-25 minutes. The questionnaire is adopted by the researcher from relevant kinds of literature considering the cultural, political, historical, and economic values of the study area.

The questionnaire was separated into four parts: socioeconomic, dietary patterns, food choice behaviors, and physical examination. Physical examination was applied to do the anthropometric measurements such as weight, height, mid-upper arm circumference (MUAC), waist circumference, waist-to-hip ratio, wrist and thigh circumference at the right side, and hip circumference, which were taken from women. Triplicate measurements were done on the same day for each study subject using calibrated equipment and standardized techniques, and the mean of the measures was reported as the final measure. 


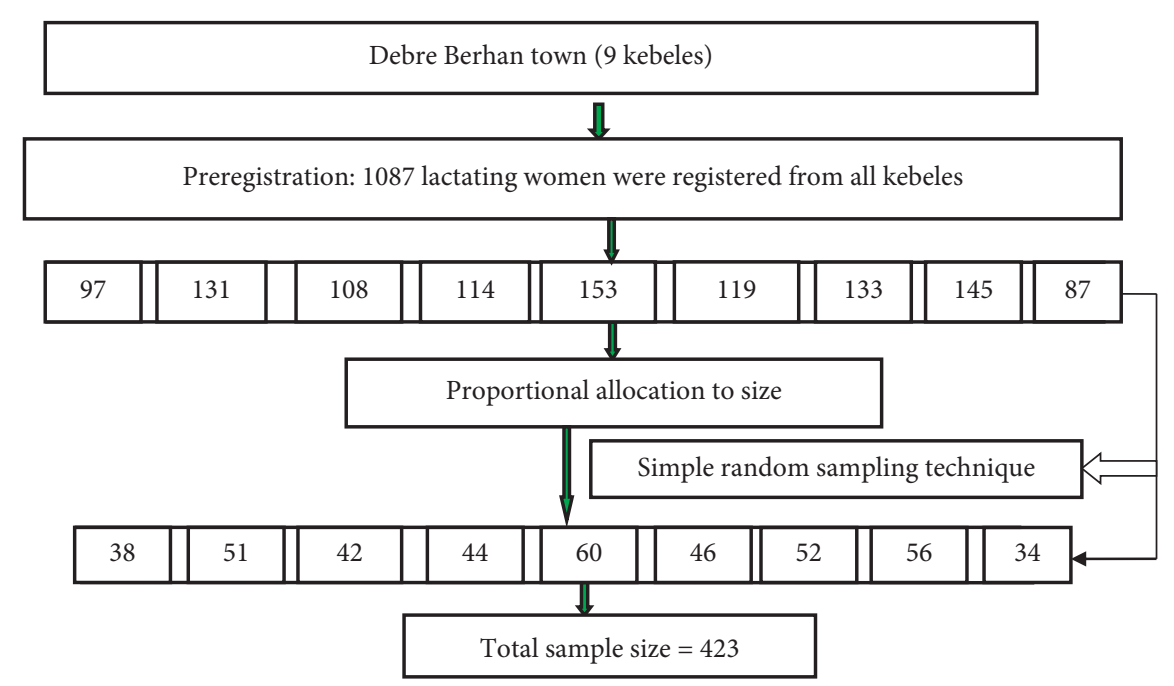

FIGURE 1: Schematic representation of sampling techniques for studied lactating women $(n=423)$ in the case of central Amhara, Ethiopia, from March to June 2016.

Furthermore, removing shoes and heavy clothes were done for weight and height measurements. Accordingly, height was measured to the nearest b0.1 cm with barefoot, and weight was measured to the nearest $0.1 \mathrm{~kg}$ with light clothing. Body mass index was obtained by dividing the weight in kilogram by the height in meter squared $\left(\mathrm{kg} / \mathrm{m}^{2}\right)$. The mid-upper arm circumference was measured by using a nonstretchable MUAC measuring tape. Then, classification was applied using the cut-off point $\mathrm{MUAC}<21 \mathrm{~cm}$ and MUAC $\geq 21 \mathrm{~cm}[32]$.

2.5. Data Quality Assurance. To address major areas of bias that can be introduced during the data collection process, the following actions were considered critically. First, the English version of the questionnaire was translated into a survey language which was Amharic and retranslated back to English for consistency by comparing with the original version. Reviewing of the questionnaire was done by senior researchers, and comments were incorporated. Then, the final version questionnaire was prepared and pretested in 22 lactating women to check its understandability by the study participants, the response rate to each question, determine the time required per questionnaire, and the necessary corrections were taken. Three days of training on the objective of the study were prepared by the principal investigator and given to data collectors on interviewing techniques and data collection tools. Site supervision, daily monitoring, and cross-checking of data for completeness and consistency of collected data were carried out by supervisors as well as the principal investigator.

Data on FVs consumption were obtained separately with 24-hour recalling method using questions adapted from the WHO [33]. One standard serving size equals 80 grams and is measured by showing pictorial show cards of common FVs found in the study setting. The picture cards were important to recalling FVs intake and minimizing error in the estimation of standard portions consumed. Medium-size fruits such as orange, apple, banana, and other fruits like half of the avocado, large mango, half cup of fruit juice, one cup of raw green vegetables, half cup for cooking (chopped) vegetables/ fruits counted as one portion size. Then, participants who had two daily serving sizes for fruits and three daily serving sizes for vegetables were merged and given a 1 value to denote the FVs consumption category which is important to determine whether each participant met the criterion [20]. The final dichotomous variables were created by classifying participants as "meets criterion-good consumption" and "does not criterion."

2.6. Data Analysis. Data was captured by the researcher using SPSS version 20 computer software program. Descriptive statistics were computed for all relevant variables (socioeconomic characteristics, FVs intake, food choice behavior, and anthropometric indices). Multicollinearity and model fitness were also checked. The nutritional status of women as a dependent variable was classified into BMI $<18.5$ and $\mathrm{BMI} \geq 18.5 \mathrm{~kg} / \mathrm{m}^{2}$. To determine the association between predicting food choice behaviors of women, logistic regression was used with both bivariate and multivariate analyses. Candidate variables were selected and transferred to a multivariate analysis by using a preset $P$-value of $<0.25$. Adjusted odds ratios with $95 \%$ confidence interval were the outcomes of logistic regression and reported to quantify the strength of associations. A $P$-value of less than 0.05 was taken as statistically significant motivational behaviors of the outcome variable.

2.7. Ethics and Consent. The objective of the study was clarified to Debre Berhan town administrative health care officials for their permission and support. The purpose of the study was explained to the study participants and written consent was taken. The responses were kept confidential by coding. Finally, nutrition information about FVs consumption and its importance was given to women in the study area. 


\section{Result}

3.1. Sociodemographic Status of Women. A total of 423 lactating women (100\% participation) were interviewed in this study. Accordingly, about $63.8 \%$ of the respondents had $26-35$ years old, $46.1 \%$ of respondents had completed higher education (college and above), $81.8 \%$ were married, and $53.9 \%$ had worked for money/payment (see Table 1). This study also examined the estimated household income earned per month from income-generating activities. The classification was done using the median of monthly income and $50.2 \%$ of the respondents earned $\leq 3500$ Ethiopian birr per month.

3.2. Anthropometrics Characteristics. About 26.5\%, 5\%, and $13.5 \%$ of women had body weight $\leq 45 \mathrm{~kg}$, height $\leq 145 \mathrm{~cm}$, and MUAC $<21 \mathrm{~cm}$. About $92.7 \%, 83.5 \%$, and $75.7 \%$ of women had a waist circumference $\leq$ of $80 \mathrm{~cm}$, waist-to-hip ratio $\leq$ of 0.8 , and the waist-to-height ratio $\leq$ of 0.5 . The prevalence of chronic energy deficiency was $21.7 \%$ with a mean value of $21.52 \pm 2.71$ (see Table 2 ).

3.3. Food Choice Behaviors. The current study provides a basic concept regarding the food choice behaviors of women. This study showed that food selection based on religion was mentioned by over 388 (91.7\%) of respondents. Accordingly, lactating women reported price 355 (83.9\%), preparation convenience $352(83.2 \%)$, best friend encouragement 344 (81.3\%), husband encouragement 300 (70.9\%), meal healthiness $280(66.2 \%)$, and daily availability of fruit and vegetable $266(62.9 \%)$ as mainly key drivers (see Table 3 ).

The perceived price of selected FVs items for those prices concerned with studied lactating women is presented in Table 4. Choosing foods based on taste, its ingredient content, for weight control, medical reason, and based ethical value was mentioned by 237 (56\%), 209 (49.1\%), 194 (45.9\%), 193 (45.6\%), and 164 (38.8\%) of study respondents, respectively. Among participants who were conscious of weight, food items low in carbohydrate and fat contents were preferred. The ethical concern during food choice signals a common area in origin, environmental acceptance, and official recognition of foods. Avoiding because of nutrient content was mentioned by 144 (34\%) of study participants. Choosing foods for mood purposes was the least underlying motives, which accounted for 92 (22\%). Choosing foods for feeling good (23.9\%) and coping with life $(21.7 \%)$ were the most important mood options next to keeping alert which accounted for 35 (38\%) (see Table 3).

3.4. Fruits and Vegetable Consumption. Good consumption of FVs was observed among $37.4 \%$ of the studied lactating women (see Table 5), i.e., the prevalence of meeting daily recommendations for daily eating of FVs. Fruits tended to be consumed less frequently than vegetables.

3.5. Association between Food Choice Behaviors and FVs Consumption. The primary objective of this study is to investigate the association between food choice behaviors
TABLE 1: Sociodemographic characteristics of lactating women $(n=423)$ in central Amhara from March to June 2016.

\begin{tabular}{|c|c|c|}
\hline Variables & Frequency $(n)$ & Percentage (\%) \\
\hline Age groups & $28.79 \pm(4.97)^{1}$ & \\
\hline $15-25$ years & 109 & 25.8 \\
\hline $26-35$ years & 270 & 63.8 \\
\hline $36-49$ years & 44 & 10.4 \\
\hline \multicolumn{3}{|l|}{ Educational status } \\
\hline No formal education & 30 & 7.1 \\
\hline Primary & 97 & 22.9 \\
\hline Secondary & 101 & 23.9 \\
\hline College and above & 195 & 46.1 \\
\hline \multicolumn{3}{|l|}{ Marital status } \\
\hline Married & 346 & 81.8 \\
\hline Other & 77 & 18.2 \\
\hline \multicolumn{3}{|l|}{ Head of a family } \\
\hline Woman & 71 & 16.78 \\
\hline Other & 352 & 83.2 \\
\hline \multicolumn{3}{|l|}{ Occupation } \\
\hline Not having work & 195 & 46.1 \\
\hline Having work & 228 & 53.9 \\
\hline Family size & $4.39 \pm 1.41$ & \\
\hline 2-4 persons & 240 & 56.7 \\
\hline$\geq 5$ persons & 183 & 43.3 \\
\hline \multicolumn{3}{|c|}{ Length of the lactation period } \\
\hline$\leq 12$ months & 233 & 55.08 \\
\hline$>13$ months & 190 & 44.92 \\
\hline \multicolumn{3}{|l|}{ Monthly income in ETB } \\
\hline$\leq 3500$ & 215 & 50.2 \\
\hline$>3500$ & 208 & 49.8 \\
\hline
\end{tabular}

Note. $1=$ mean \pm standard deviation. ETB $=$ Ethiopian Birr.

TABLE 2: Anthropometric characteristics of studied women in central Amhara from March to June $2016(n=423)$.

\begin{tabular}{lcc}
\hline Variables & Frequency $(n)$ & Percentage $(\%)$ \\
\hline Weight in $\mathrm{kg}$ & & \\
$\quad \leq 45$ & 112 & 26.5 \\
$>45$ & 311 & 73.5 \\
\hline Height in cm & & \\
$\quad \leq 145$ & 21 & 5 \\
$>145$ & 402 & 95 \\
\hline MUAC in cm & & \\
$<21$ & 57 & 13.5 \\
$\geq 21$ & 366 & 86.5 \\
\hline Waist circumference in cm & \\
$\leq 80$ & 392 & 92.7 \\
$>80$ & 31 & 7.3 \\
\hline Waist-to-hip ratio & & \\
$\leq 0.8$ & 353 & 83.5 \\
$>0.8$ & 70 & 16.5 \\
\hline Waist-to-height ratio & & \\
$\leq 0.5$ & 320 & 75.7 \\
$>0.5$ & 103 & 24.3 \\
\hline BMI in kg/m & & \\
$<18.49$ & 92 & 69.5 \\
$18.5-24.99$ & 294 & 8.8 \\
$\geq 25$ & 37 & \\
\hline
\end{tabular}

Note. $\mathrm{MUAC}=$ mid-upper arm circumference, $\mathrm{BMI}=$ body mass index, $\mathrm{cm}=$ centimeter, and $\mathrm{kg}=$ kilogram . 
TABLE 3: Food choice behaviors among lactating women in central Amhara from March to June $2016(n=423)$.

\begin{tabular}{|c|c|c|c|}
\hline Variables & $n$ & $\%$ & $95 \% \mathrm{CI}$ \\
\hline \multicolumn{4}{|l|}{ Healthy value of a diet } \\
\hline Yes & 280 & 66.2 & $61.5-71.7$ \\
\hline Vitamin/mineral & 128 & 45.71 & \\
\hline Keeping me healthy & 77 & 27.5 & \\
\hline Reduce adverse risk & 15 & 5.36 & \\
\hline High in protein & 43 & 15.36 & \\
\hline Good for skin, teeth, hair, and nail & 2 & 0.71 & \\
\hline Recommended by physicians & 15 & 5.36 & \\
\hline No & 143 & 33.8 & $28.3-38.5$ \\
\hline \multicolumn{4}{|l|}{ Avoiding nutrient content } \\
\hline Yes & 144 & 34 & $29.5-39.2$ \\
\hline High in fat & 75 & 52.08 & \\
\hline High in $\mathrm{CHO}$ & 49 & 34.03 & \\
\hline High in $\mathrm{CHO}$ and fat & 4 & 2.78 & \\
\hline High in salt & 7 & 4.86 & \\
\hline Pungent pepper-containing foods & 9 & 6.25 & \\
\hline No & 279 & 66 & $60.8-70.4$ \\
\hline \multicolumn{4}{|l|}{ Ingredient content } \\
\hline Yes & 209 & 49.1 & $44.8-54.1$ \\
\hline Natural ingredients & 134 & 64.1 & \\
\hline Artificial ingredients & 75 & 35.9 & \\
\hline No & 214 & 50.6 & $45.1-55.2$ \\
\hline \multicolumn{4}{|l|}{ Price concern } \\
\hline Yes & 355 & 83.9 & $80-87.5$ \\
\hline No & 68 & 16.1 & $12.5-6-20$ \\
\hline \multicolumn{4}{|l|}{ Taste } \\
\hline Yes & 237 & 56 & $51.3-60.6$ \\
\hline No & 186 & 44 & $39-48.7$ \\
\hline \multicolumn{4}{|l|}{ Mood } \\
\hline Yes & 92 & 22 & $18-25.3$ \\
\hline Helps me cope with stress & 20 & 21.74 & \\
\hline Helps me cope with life & 2 & 2.17 & \\
\hline Helps me relax & 11 & 11.96 & \\
\hline Keeping me alert & 35 & 38.04 & \\
\hline Cheering me up & 2 & 2.17 & \\
\hline Helps me feel good & 22 & 23.91 & \\
\hline No & 331 & 78 & $74.7-82$ \\
\hline \multicolumn{4}{|l|}{ Preparation convenience } \\
\hline Yes & 352 & 83.2 & $79.7-86.5$ \\
\hline No & 71 & 16.8 & $13.5-20.3$ \\
\hline \multicolumn{4}{|l|}{ Religion taboo } \\
\hline Yes & 388 & 91.7 & $89.1-94.3$ \\
\hline No & 35 & 54.4 & $5.7-10.9$ \\
\hline \multicolumn{4}{|l|}{ Weight control } \\
\hline Yes & 194 & 45.9 & $41.6-50.4$ \\
\hline Low in carbohydrate & 71 & 36.6 & \\
\hline Help me to control weight & 47 & 24.23 & \\
\hline Helps me to admire people & 3 & 1.55 & \\
\hline Helps me have a smart weight & 6 & 3.09 & \\
\hline Low in fat & 67 & 34.54 & \\
\hline No & 229 & 54.1 & $49.6-59.4$ \\
\hline \multicolumn{4}{|l|}{ Health concern (medical reason) } \\
\hline Yes & 193 & 45.6 & $41.1-50.4$ \\
\hline No & 230 & 54.4 & $49.6-58.9$ \\
\hline \multicolumn{4}{|l|}{ Worry about their health } \\
\hline Gastric disease & 112 & & \\
\hline Heart case & 21 & & \\
\hline Diabetes & 24 & & \\
\hline Kidney & 6 & & \\
\hline Allergy & 7 & & \\
\hline Blood pressure & 11 & & \\
\hline Cancer & 12 & & \\
\hline
\end{tabular}


TABLE 3: Continued.

\begin{tabular}{|c|c|c|c|}
\hline Variables & $n$ & $\%$ & $95 \% \mathrm{CI}$ \\
\hline \multicolumn{4}{|c|}{ Peer pressure } \\
\hline Yes & 344 & 81.3 & $77.3-85.6$ \\
\hline No & 79 & 18.7 & $14.4-22.7$ \\
\hline \multicolumn{4}{|c|}{ Influence of husband } \\
\hline Yes & 300 & 86.7 & $82.8-89.9$ \\
\hline No & 46 & 13.3 & $10.1-17.2$ \\
\hline \multicolumn{4}{|c|}{ Availability of FVs at working place } \\
\hline Yes & 95 & 33.8 & $28.3-39.5$ \\
\hline No & 186 & 66.2 & $60.5-71.7$ \\
\hline \multicolumn{4}{|c|}{ Availability of FVs at home } \\
\hline Yes & 266 & 62.9 & $57.4-67.1$ \\
\hline No & 157 & 37.1 & $32.9-42.6$ \\
\hline \multicolumn{4}{|c|}{ Availability of soft drink at home } \\
\hline Yes & 147 & 34.8 & $30.3-39.7$ \\
\hline No & 276 & 65.2 & $60.3-69.7$ \\
\hline \multicolumn{4}{|c|}{ Availability of dairy products at home } \\
\hline Yes & 317 & 74.9 & $70.7-79$ \\
\hline No & 106 & 25.1 & $21-29.3$ \\
\hline
\end{tabular}

and FVs intake. As a result, to investigate food choice behaviors influencing the FVs consumption of the women, logistic regression analysis was used using both bivariate and multivariate analyses. As a result, healthy meal, ingredient content, avoiding nutrient content, mood, religious influence, husband encouragement, and availability of (FVs and milk and milk products) at the home were significantly associated with the FVs consumption of lactating women during bivariate analysis $(P<0.05)$ (see Table 6$)$.

After adjusting for all candidate variables (preference for a healthy diet, to avoid some nutrient, mood, nutrient content, convenience, price, medical reason, weight control, religion taboo, peer pressure, the influence of the husband, availability of FVs at home, and availability of dairy products at home) in the multivariate logistic analysis; natural content, mood concern, religious influence, husband encouragement, and availability of FVs and milk and milk products were associated with FVs consumption $(P<0.05)$ (see Table 6). Accordingly, among lactating women who were highly motivated to choose a meal based on natural content, mood concern, religious influence, and encouragement by their husband, FVs consumption was significantly higher with AOR (95\%) 2.37 (1.39-4.06), 2.29 $(1.15-4.56), 2.45$ (1.37-4.4), 2.26 (1.04-4.89), and 1.66 (1.16-3.48), respectively.

3.6. Association of Food Choice Behaviors and Nutritional Status among Women. Healthy meal motivation, mood, and price concern were significantly associated with the nutritional status of women during bivariate analysis $(P<0.05)$ (see Table 7). After adjusting for all candidate variables in multivariate analysis, healthy meals, price, and mood concern were associated significantly with the nutritional status of women $(P<0.05)$ (see Table 7$)$. Accordingly, for lactating women who were motivated to choose healthy meals and price concerns, normal weight was significantly higher with an AOR of 2.1 (95\% CI: 1.21-3.62) and 3.01 (95\% CI: 1.32-6.9), respectively. Compared to lactating women, who were concerned to choose food for mood purposes, normal weight was significantly lower among women who were not concerned with an AOR of 0.5 (95\% CI: 0.30-0.95).

\section{Discussion}

The present study discusses the nutritional status and FVs intake behaviors of lactating women. Furthermore, as a principal finding, the study also elaborates the influencing contribution of food choice behaviors on nutritional status and FVs intake of women during lactation from central Ethiopia. Accordingly, the FVs consumption trend and nutritional status were determined. As a result, about $37.4 \%$ of lactating women had good trends in consuming FVs. However, this finding was higher compared to other studies done in Ethiopia [34], Uganda [35], and Atlanta [36] with a prevalence of 20, 12.7\%, and $27.7 \%$, respectively. The prevalence of body mass index less than $18.5 \mathrm{~kg} / \mathrm{m}^{2}$ among women was $21.7 \%$, which was lower than the finding of Tigray region of Ethiopia (25\%) [7], and the Ethiopian Health and Nutrition Research Institute nutrition baseline survey report (28.8\%) [37].

Furthermore, the prevalence was much lower than the result of other studies [6, 38, 39], which was about $37.5 \%$. Perhaps, the community tested might be accessible to various health promotions and the kind of nutrition that they would require to maintain their health due to the given time interval. It is also analogous to a study done by Haregu and his colleagues [40].This present study showed that vegetable consumption was higher compared to fruit consumption, which is similar to a study done in SNNPR by Handiso, but the prevalence is still lower [41]. This might be because women from the southern region have access to those agricultural products. However, the prevalence of this finding is not in agreement with a study done by Chen and Gazmararian (2014), which showed low consumption of green salad, carrot, and other vegetables than fruit juice and fruit intake [40].

The main finding of this study was that Debre Berhan women's FVs consumption and nutritional status were 


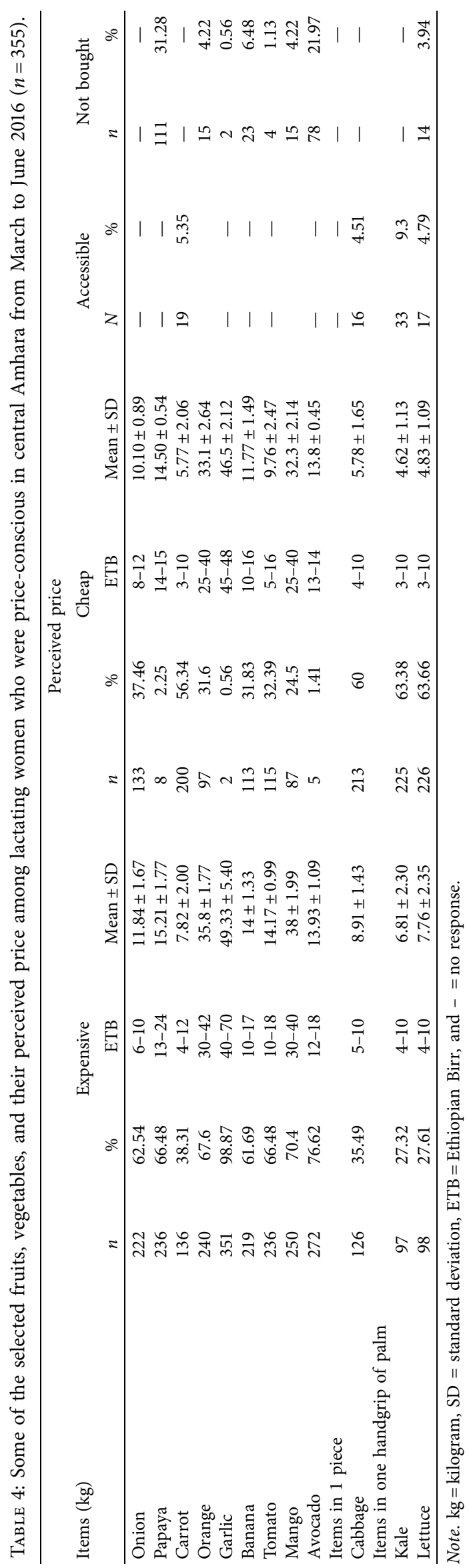


Table 5: Prevalence of FVs intake among lactating women in central Amhara from March to June $2016(n=423)$.

\begin{tabular}{|c|c|c|c|c|}
\hline \multirow{3}{*}{ Daily intake trend in average } & \multicolumn{4}{|c|}{ Response } \\
\hline & \multicolumn{2}{|c|}{ Yes } & \multicolumn{2}{|c|}{ No } \\
\hline & $n$ & $\%$ & $n$ & $\%$ \\
\hline Vegetables (3 servings) & 218 & 51.54 & 205 & $\overline{48.46}$ \\
\hline Fruits ( 2 servings) & 161 & 38.06 & 262 & 61.94 \\
\hline Fruits and vegetables ( 5 servings) & 158 & 37.4 & 265 & 62.65 \\
\hline
\end{tabular}

TABLE 6: The association with FVs intake among lactating women in central Amhara from March to June 2016 ( $n=423)$.

\begin{tabular}{lcc}
\hline Food choice behaviors & COR $(\mathrm{CI})$ & The choice for FVs intake \\
\hline Preference for a healthy diet & $1.91(1.27-2.89)^{* *}$ & $1.58(0.91-2.77)^{*}$ \\
To avoid some nutrient & $1.73(1.12-2.66)^{* *}$ & $1.68(0.97-2.91)^{*}$ \\
Mood & $2.07(1.23-3.48)^{* *}$ & $2.29(1.15-4.56)^{* *}$ \\
Taste & $1.11(0.75-1.65)$ & $2.37(1.39-4.06)^{* *}$ \\
Nutrient content & $3.19(2.11-4.83)^{* *}$ & $1.06(0.51-2.19)$ \\
Convenience & $1.58(0.94-2.64)^{*}$ & $0.61(0.29-1.26)^{*}$ \\
Price & $0.6(0.34-1.07)^{*}$ & $0.85(0.51-1.4)$ \\
Medical reason & $1.05(0.7-1.56)^{*}$ & $0.96(0.58-1.59)$ \\
Weight control & $1.3(0.88-1.94)^{*}$ & \\
Ethics & $1.01(0.68-1.52)$ & $2.45(1.37-4.4)^{* *}$ \\
Religion taboo & $1.81(1.18-2.79)^{* *}$ & $1.12(0.58-2.14)^{*}$ \\
Peer pressure & $1.62(0.99-2.66)^{*}$ & $2.26(1.04-4.89)^{* *}$ \\
Influence of husband & $2.81(1.49-5.28)^{* *}$ & $1.04(0.75-2.26)$ \\
Availability of FVs at working place & $1.3(1.53-3.45)^{* *}$ & $1.66(1.16-3.48)^{* *}$ \\
Availability of FVs at home & $2.13(1.36-3.33)^{* *}$ & $(1.39-2.74)^{* *}$ \\
Availability of dairy products at home & & 1.6 \\
\hline
\end{tabular}

Note. $\mathrm{FVs}=$ fruits and vegetables, ${ }^{* *}=P<0.05,{ }^{*} \mathrm{P} \leq 0.25, \mathrm{COR}=$ crude odds ratios, $\mathrm{AOR}=$ adjusted odds ratio, and $\mathrm{CI}=$ confidence interval.

associated with food choice behaviors. Among lactating women who were highly motivated to choose a meal for natural content, meals made from natural ingredients were more preferable and perceived to be healthy, including porridge, brown bread, fruits, and vegetables [42]. The likelihood of being in the normal weight among lactating women was directly associated with strong healthy eating motivation. The strong association between healthy meals eating motivation and nutritional status might be explained by the fact that those who had higher motivation had increased and diversified food consumption as compared to those who had no motivation. Since people's motivations towards eating a healthy diet are generally positive to obtain better quality diets which can affect their nutritional status. The finding was inconsistent with Naughton et al.'s study [31].

FVs consumption was significantly higher among women who were concerned about their mood. It contradicted the result explained as emotional eaters consume sweet, fatty foods to regulate their emotions while it was unrelated to the consumption of FVs [43-45]. This could be due to the variation among current and past studied target population. Women encouraged by their husbands had higher FVs consumption. The possible explanation could be social support from within the household and from coworkers was positively associated with improvements in FVs consumption. Because family and friends can be a source of encouragement in making and sustaining dietary change, adopting dietary strategies that are acceptable to them may benefit the individual whilst also having an effect on the eating habits of others [28]. The prevalence of underweight was significantly higher among women who were concerned about stress during food choice. This could suggest that those study participants are affected by their emotions to choose different food items. Psychological stress is a common feature of life and can modify eating behaviors leading to poor quality of food consumption due to mental stress initiated by working under time pressure during peak periods. This increases the risk of poor nutritional status and ultimately jeopardizing their health prognosis and related outcomes [27]. Research has shown that along with easier access and availability of unhealthy foods, bodyweight statuses are closely associated with tension and anxiety within the workplace [46].

Women who were highly concerned with their religion had good FVs consumption since FVs are fasting food groups and preferable for fasting time especially for Orthodox religion which is practiced among studied women. Additionally, FVs consumption was significantly higher among lactating women with home availability of FVs. The availability of healthy food at home and away from home in the community increases the consumption of such foods [47]. FVs consumption was significantly higher among lactating women with home availability of milk and milk products. This could be due to the complementary behavior 
TABLE 7: The food choice behaviors associated with the nutritional status among lactating women in central Amhara from March to June $2016(n=423)$.

\begin{tabular}{|c|c|c|c|c|}
\hline \multirow[b]{2}{*}{ Variables } & \multicolumn{4}{|c|}{ Nutritional status } \\
\hline & $\begin{array}{l}\text { Undernourished } \\
\left(\mathrm{BMI}<18.5 \mathrm{~kg} / \mathrm{m}^{2}\right)\end{array}$ & $\begin{array}{c}\text { Normal } \\
\left(\mathrm{BMI} \geq 18.5 \mathrm{~kg} / \mathrm{m}^{2}\right)\end{array}$ & $\mathrm{COR}(\mathrm{CI})$ & $\mathrm{AOR}(\mathrm{CI})$ \\
\hline \multirow[t]{2}{*}{ Avoiding food as not good for a child } & 59 & 233 & $0.96(0.58-1.61)$ & \\
\hline & 35 & 96 & & \\
\hline \multirow[t]{2}{*}{ Healthy meal } & 71 & 209 & $1.97(1.156-3.37)^{* *}$ & $2.1(1.21-3.62)^{* *}$ \\
\hline & 21 & 122 & & \\
\hline \multirow[t]{2}{*}{ Avoid nutrient } & 30 & 114 & $0.92(0.56-1.51$ & \\
\hline & 62 & 217 & & \\
\hline \multirow[t]{2}{*}{ Mood } & 13 & 79 & $0.53(0.28-0.99)^{* *}$ & $0.5(0.30-0.95)^{* *}$ \\
\hline & 79 & 252 & & \\
\hline \multirow[t]{2}{*}{ Taste } & 50 & 187 & $0.92(0.58-1.46)$ & \\
\hline & 42 & 144 & & \\
\hline \multirow[t]{2}{*}{ Natural content } & 164 & 167 & $0.79(0.5-1.26)$ & \\
\hline & 51 & 41 & & \\
\hline \multirow[t]{2}{*}{ Preparation convenience } & 73 & 279 & $0.72(0.4-1.29)$ & \\
\hline & 19 & 52 & & \\
\hline \multirow[t]{2}{*}{ Price } & 85 & 270 & $2.74(1.21-6.22)^{* *}$ & $3.01(1.32-6.9)^{* *}$ \\
\hline & 7 & 61 & & \\
\hline \multirow[t]{2}{*}{ Medical reason } & 36 & 157 & $0.71(0.45-1.14)^{*}$ & $0.65(0.40-1.06)$ \\
\hline & 56 & 174 & & \\
\hline \multirow[t]{2}{*}{ Weight } & 40 & 154 & $0.88(0.56-1.41)$ & \\
\hline & 52 & 177 & & \\
\hline \multirow[t]{2}{*}{ Religion influence } & 87 & 301 & $1.73(0.65-4.6)$ & \\
\hline & 5 & 30 & & \\
\hline \multirow[t]{2}{*}{ Friend encouragement } & 72 & 272 & $0.78(0.44-1.38)$ & \\
\hline & 20 & 59 & & \\
\hline \multirow[t]{2}{*}{ Husband encouragement } & 44 & 256 & $0.49(0.23-1.02)^{*}$ & $0.81(0.33-2.86)$ \\
\hline & 12 & 34 & & \\
\hline \multirow[t]{2}{*}{ Availability of soft drink at home } & 32 & 115 & $0.46(0.25-0.86)^{* *}$ & $0.47(0.2-1.14)^{*}$ \\
\hline & 60 & 215 & & \\
\hline \multirow[t]{2}{*}{ Availability of FVs at home } & 54 & 212 & $0.8(0.5-1.28)$ & \\
\hline & 38 & 119 & & \\
\hline \multirow[t]{2}{*}{ Availability of milk and milk products at home } & 64 & 253 & $0.71(0.42-1.18)^{*}$ & $2.4(0.67-8.66)^{*}$ \\
\hline & 28 & 78 & & \\
\hline \multirow[t]{2}{*}{ Advertisement } & 30 & 104 & $1.1(0.65-1.73)$ & \\
\hline & 62 & 227 & & \\
\hline \multirow[t]{2}{*}{ Nutrition books, journals, and magazines } & 22 & 73 & $1.11(0.64-1.92)$ & \\
\hline & 70 & 258 & & \\
\hline
\end{tabular}

Note. $\mathrm{FVs}=$ fruits and vegetables, ${ }^{* *} \mathrm{P} \leq 0.05,{ }^{*} \mathrm{P} \leq 0.25, \mathrm{AOR}=$ adjusted odd ratio, $\mathrm{BMI}=$ body mass index $\mathrm{CI}=$ confidence interval, and $\mathrm{COR}=\mathrm{crude}$ odds ratio.

of women concerning the selection decision of such healthy food groups.

Furthermore, price-concerned lactating women were more likely to be normal in their body weight. It is comparable to the study of Epstein et al., which described the relation of cross-price elasticity with bodyweight [48]. This result suggests that leaner mothers may be more likely to shift purchasing from low energy-dense to high energy-dense foods based on price changes of low energydense foods. Other expected reasons could be the purchasing power of women may be limited to the specific food items and influenced by the purchasing frequencies of food items. Additionally, the knowledge of study respondents may affect price over income, i.e., using available resources wisely to choose a balanced diet may be predominated in those lactating women. The less expected association between meal healthy value consciousness and FVs consumption was insignificant and inconsistent with previous studies showing a significant association involving higher FVs consumption and healthy eating motivation $[31,49,50]$.

Strengths and Weaknesses. The community-based approach, random selection of the study population, and the study samples were selected directly from the targeted population and an interviewer was present, and respondents answering questions about their chosen task were the major strengths of the current study. This may allow generalization since an attempt was made to identify randomized lactating women from the target communities. Since the study consisted of only lactating women, the results may not apply to the general population who were not lactating women. Even if 
the sample size was considerable, due to resource scarcity, the study respondents were limited to 423 participants. Still, miscounting of eligible women and other biases like social desirability, selection, interviewer, measurement, and information bias could be expected. Particularly, the introduction of partiality is expected during the anthropometric measurement and FVs intake assessment due to measurement and interviewer bias, respectively. Recalling bias, overestimation, and underestimation of food items could be expected at the data collection process. Literature review and discussion were done with other target groups from other countries that might have different settings and this could make a great variation. A limitation of a survey questionnaire is that one can only include and compare a limited number of attributes. Furthermore, cross-sectional data is a limit within itself to determine cause or effect. It relatively indicates a snapshot of a moment in time of the person's daily life.

\section{Conclusions and Recommendation}

FVs consumption and normal weight of women were assessed and it is low in this study. Furthermore, food choice behaviors associated with the consumption of FVs and nutritional status were identified. Accordingly, ingredient content, mood concern, religious concern, and home availability (of milk and milk products and FVs) were associated significantly with FVs intake. Additionally, healthy meal motivation, price, and mood concern were the most important food choice behaviors associated with the nutritional status of lactating women. This research is useful to create insight about food choice behaviors for a group of women with their motivations and lead practitioners to be able to make a recommendation as to what health promotion strategies one should be based on.

$\begin{array}{ll}\text { Abbreviations } \\ \text { AOR: } & \text { Adjusted odds ratio } \\ \text { BMI: } & \text { Body mass index } \\ \text { CI: } & \text { Confidence interval } \\ \text { Cm: } & \text { Centimeter } \\ \text { COR: } & \text { Crude odds ratio } \\ \text { CSA: } & \text { Central Statistics Agency } \\ \text { EDHS: } & \text { Ethiopia Demographic and Health Survey } \\ \text { EMDHS: } & \text { Ethiopia Mini Demographic and Health Survey } \\ \text { ETB: } & \text { Ethiopian birr } \\ \text { FAO: } & \text { Food and Agriculture Organization } \\ \text { FMOH: } & \text { Federal Minister of Health } \\ \text { FVs: } & \text { Fruits and vegetables } \\ \text { Kg: } & \text { Kilogram } \\ \text { NCDs: } & \text { Noncommunicable diseases } \\ \text { SPSS: } & \text { Statistical Package for Social Sciences } \\ \text { SD: } & \text { Standard deviation } \\ \text { UNICEF: } & \text { United Nations International Children's } \\ & \text { Emergency Fund } \\ \text { USAID: } & \text { United States Agency for International } \\ \text { WHO: } & \text { Development } \\ & \text { World Health Organization. }\end{array}$

\section{Data Availability}

The datasets used and analyzed to support the findings of this study are available from the corresponding author upon reasonable request.

\section{Consent}

The purpose of the study was explained to the study participants and written informed consent was taken.

\section{Conflicts of Interest}

The authors declare that they have no conflicts of interest regarding the publication of this paper.

\section{Authors' Contributions}

GK developed the study, design, implementation, analysis, interpretation, and final writing.

\section{Acknowledgments}

The author wants to express gratitude to the data collectors and study participants for their support in this study. This work was supported by Innovation and Technology Transfer Grants from Wollega University.

\section{References}

[1] R. E. Black, L. H. Allen, Z. A. Bhutta et al., "Maternal and child undernutrition: global and regional exposures and health consequences," The Lancet, vol. 371, no. 9608, pp. 243-260, 2008.

[2] M. S. Jan and E. S. Tee, "Changing trends in dietary pattern and implications to food and nutrition security in the association of southeast asian nations," International Journal of Nutrition and Food Sciences, vol. 3, no. 4, pp. 259-269, 2014.

[3] G. Ruel, Z. Shi, S. Zhen et al., "Association between nutrition and the evolution of multimorbidity: the importance of fruits and vegetables and whole grain products," Clinical Nutrition, vol. 33, no. 3, pp. 513-520, 2014.

[4] G. Kibr, "Nutritional status and associated drivers of food choice among lactating women in Debre Birhan Town, North Shoa Zone, Amhara Region, Ethiopia: a community based cross-sectional study," International Journal of Nutrition and Metabolism, vol. 12, no. 2, pp. 16-24, 2020.

[5] M. H. Forouzanfar, L. Alexander, and H. R. Anderson, "Global, regional, and national comparative risk assessment of behavioral, environmental and occupational, and metabolic risks or clusters of risks in 188 countries, 1990-2013: a systematic analysis for the Global Burden of Disease Study," Lancet, vol. 386, pp. 2287-2323, 2013.

[6] T. Nega, "Dietary adequacy and nutritional practice of lactating mothers in wonsho woreda sidama zone southern Ethiopia," MSc. Thesis, College of Agriculture, Hawassa University, Hawassa. Ethiopia, 2010.

[7] K. Haileslassie, A. Mulugeta, and M. Girma, "Feeding practices, nutritional status, and associated factors of lactating women in Samre Woreda, South Eastern Zone of Tigray, Ethiopia," Nutrition Journal, vol. 12, p. 28, 2013.

[8] D. H. Temesgen, F. G. Habtamu, D. W. Demeda, and N. K. Dunkana, "Nutritional status, and associated factors 
among Lactating Mothers in Nekemte Referral hospital and health center, Ethiopia," Food Science and Quality Management, vol. 35, pp. 64-72, 2015.

[9] H. H. Tikuye, S. Gebremedhin, A. Mesfin, and S. J. Whiting, "Prevalence and factors associated with undernutrition among exclusively breastfeeding women in arba minch zuriya district, southern Ethiopia: a cross-sectional communitybased study," Ethiopian Journal of Health Sciences, vol. 28, no. $5,2019$.

[10] World Health Organization, Africa Regional Health Office: The Health of the People. Africa Regional Health Report, World Health Organization, Geneva, Switzerland, 2007.

[11] Federal Ministry of Health, Program Implementation Manual of National Nutrition Program, Addis Ababa, Ethiopia, 2008.

[12] Central Statistics Agency, Ethiopia Demographic and Health Survey, Central Statistics Agency, Addis Ababa, Ethiopia, 2011.

[13] Central Statistics Agency, Ethiopia Mini Demographic and Health Survey, Central Statistics Agency, Addis Ababa, Ethiopia, 2014.

[14] United States Agency for International Development, Ethiopia Nutrition Profile, United States Agency for International Development, Washington, D.C., USA, 2014.

[15] Central Statistical Agency, "Federal democratic republic of Ethiopia: household income, consumption, and expenditure survey of 2004/5," Addis Ababa: Statistical Bulletin, vol. 394, p. 1, 2007.

[16] M. Prevett, "Chronic non-communicable diseases in Ethiopia-a hidden burden," Ethiopia Journal of Health Science, vol. 22, p. 2, 2012.

[17] A. Yohannes, M. Awoke, and D. Amare, "The impact of dietary risk factors on the burden of non-communicable diseases in Ethiopia: findings from the Global Burden of Disease study," International Journal of Behavioral Nutrition and Physical Activity, vol. 13, p. 12, 2016.

[18] M. Fantahun and G. Degu, "Burden of disease in Amhara region, Ethiopia," Ethiopian Medicine Journal, vol. 42, no. 3, pp. 165-172, 2004.

[19] I. Lora, C. Kenda, and R. Marie, Improving Diet Quality and Micronutrient Nutrition, International Food Policy Research Institute, Washington, D.C., USA, 2009.

[20] WHO/FAO, Joint WHO/FAO Workshop on Fruit and Vegetables for Health, WHO Centre for Health Development, Kobe, Japan, 2004.

[21] N. P. Uusiku, A. Oelofse, K. G. Duodu, M. J. Bester, and M. Faber, "Nutritional value of leafy vegetables of subSaharan Africa and their potential contribution to human health: a review," Journal of Food Composition and Analysis, vol. 23, no. 6, pp. 499-509, 2010.

[22] M. E. Banwat, L. A. Lar, J. Daber, S. Audo, and S. Lassa, "Knowledge, and intake of fruit and vegetable consumption among adults in an urban community in North Central, Nigeria," The Nigeria Health Journal, vol. 12, no. 1, pp. 12-15, 2012.

[23] T. Pohjanheimo, "Sensory, and non-sensory factors behind the liking and choice of healthy food products western finland consumer," 2010.

[24] B. K. Thompson, M. Peck, and K. T. Brandert, "Integrating preconception health into public health practice: a tale of three cities," Journal of Women's Health, vol. 17, no. 5, pp. 723-727, 2008.

[25] K. A. Bartley, B. A. Underwood, and R. J. Deckelbaum, "A life cycle micronutrient perspective for women's health,"
American Journal of Clinical Nutrition, vol. 81, no. 1, pp. 188S-193S, 2008.

[26] M.-K. Moos, A. L. Dunlop, B. W. Jack et al., "Healthier women, healthier reproductive outcomes: recommendations for the routine care of all women of reproductive age," American Journal of Obstetrics and Gynecology, vol. 199, no. 6, pp. S280-S289, 2008.

[27] Y. Dunneram and R. Jeewon, "Healthy diet and nutrition education program among women of reproductive age: a necessity of multilevel strategies or community responsibility," Health Promotion Perspectives, vol. 5, no. 2, pp. 116-127, 2015.

[28] H. M. Inskip, S. R. Crozier, K. M. Godfrey, S. E. Borland, C. Cooper, and S. M. Robinson, "Women's compliance with nutrition and lifestyle recommendations before pregnancy: general population cohort study," The Bmj, vol. 338, no. 2, p. b481, 2009.

[29] A. Saba, M. Gebremeskel, and M. Ashenafi, "Magnitude, and chronic energy deficiency and its associated factors among women of reproductive age in Kunama population, Tigray," BMC Nutrition, vol. 1, p. 12, 2014.

[30] Central statistical agency of Ethiopia, Ethiopia Federal Democratic Republic of Ethiopia: Population Projection, Central statistical agency of Ethiopia, Addis Ababa, Ethiopia, 2015.

[31] P. Naughton, N. M. Sinéad, and B. M. Mary, "The creation of a healthy eating motivation score and its association with food choice and physical activity in a cross-sectional sample of Irish adults," International Journal of Behavioral Nutrition and Physical Activity, vol. 12, p. 74, 2015.

[32] C. Bruce, "Anthropometric indicators measurement guide," in Food and Nutrition Technical Assistance ProjectAcademy for Educational Development, Washington DC, USA, 2001.

[33] World Health Organization, "Diet, nutrition, and the prevention of chronic diseases," Technical Report Series, World Health Organization, Geneva, Switzerland, 2003.

[34] T. Marie, M. Nicholas, and S. Lisa, "Pattern's determinants of fruits and vegetable consumption in sub-saharan african," 2003.

[35] G. A. Murphy, G. Asiki, and K. Ekoru, "Socio-demographic distribution of non-communicable disease risk factors in rural Uganda: a cross-sectional study," International Journal of Epidemiology, vol. 42, no. 6, pp. 1740-1753, 2011.

[36] D. Y. Chen and J. A. Gazmararian, "Impact of personal preference and motivation on fruit and vegetable consumption of WIC-participating mothers and children in Atlanta," Journal of Nutrition Education and Behavior, vol. 46, p. 1, 2014.

[37] Ethiopian Health and Nutrition Research Institute, Nutrition Base Line Survey Report for the National Nutrition Program of Ethiopia, Ethiopian Health and Nutrition Research Institute, Addis Ababa, Ethiopia, 2008.

[38] I. E. Ransom and K. L. Elder, Nutrition of Women and Adolescent Girls: Why it Matters?, Population Reference Bureau, Washington, D.C, USA, 2003.

[39] Central Statistical Agency and ORC Macro, "Ethiopia demographic and health survey," Central statistics agency, vol. 143 , p. 164, 2006.

[40] T. N. Haregu, S. Oti, T. Egondi, and C. Kyobutungi, "Cooccurrence of behavioral risk factors of common non-communicable diseases in urban slum dwellers in Nairobi, Kenya," Global Health Action, vol. 8, no. 1, pp. 1-8, 2015.

[41] Y. H. Handiso, "Prevalence of food aversion, craving, and pica during pregnancy and their association with the nutritional 
status of pregnant women in dale Woreda Sidama zone, Ethiopia," International Journal of Nutrition and Metabolism, vol. 7, no. 1, pp. 1-14, 2014.

[42] M. Delaney and M. McCarthy, "Food choice and health across the life course: a study examining food choice in older irish adults," 2009.

[43] M. Macht, "How emotions affect eating: a five-way model," Appetite, vol. 50, no. 1, pp. 1-11, 2008.

[44] H. Konttinen, S. Männistö, S. Sarlio-Lähteenkorva, K. Silventoinen, and A. Haukkala, "Emotional eating, depressive symptoms and self-reported food consumption. A population-based study," Appetite, vol. 54, no. 3, pp. 473-479, 2010.

[45] C. Hartmann, S. Dohle, and M. Siegrist, "Time for change? Food choices in the transition to cohabitation and parenthood," Public Health Nutrition, vol. 17, no. 12, pp. 2730-2739, 2014.

[46] N. Nishitani and H. Sakakibara, "Relationship of BMI increase to eating behavior \& job stress in a 2-year cohort study of nonobese male Japanese workers," Obesity Research and Clinical Practice, vol. 1, pp. 179-185, 2005.

[47] L. Hallström, C. A. Vereecken, J. R. Ruiz et al., "Breakfast habits and factors influencing food choices at breakfast in relation to socio-demographic and family factors among European adolescents. The HELENA Study," Appetite, vol. 56, no. 3, pp. 649-657, 2011.

[48] L. H. Epstein, K. K. Dearing, R. A. Paluch, J. N. Roemmich, and D. Cho, "Price and maternal obesity influence purchasing of low- and high-energy-dense foods," The American Journal of Clinical Nutrition, vol. 86, no. 4, pp. 914-922, 2007.

[49] M. A. Beydoun and Y. Wang, "How do socio-economic status, perceived economic barriers and nutritional benefits affect quality of dietary intake among US adults?" European Journal of Clinical Nutrition, vol. 62, no. 3, pp. 303-313, 2008.

[50] K. Miura and G. Turrell, "Contribution of psychosocial factors to the association between socioeconomic position and takeaway food consumption," 2014. 